\title{
Concurrent transcriptional profiling of Dirofilaria immitis and its Wolbachia endosymbiont throughout the nematode life cycle reveals coordinated gene expression
}

Ashley N Luck', Christopher C Evans², Molly D Riggs², Jeremy M Foster ${ }^{1}$, Andrew R Moorhead², Barton E Slatko ${ }^{1}$ and Michelle L Michalski ${ }^{3^{*}}$

\begin{abstract}
Background: Dirofilaria immitis, or canine heartworm, is a filarial nematode parasite that infects dogs and other mammals worldwide. Current disease control relies on regular administration of anthelmintic preventives, however, relatively poor compliance and evidence of developing drug resistance could warrant alternative measures against D. immitis and related human filarial infections be taken. As with many other filarial nematodes, D. immitis contains Wolbachia, an obligate bacterial endosymbiont thought to be involved in providing certain critical metabolites to the nematode. Correlations between nematode and Wolbachia transcriptomes during development have not been examined. Therefore, we detailed the developmental transcriptome of both D. immitis and its Wolbachia (wDi) in order to gain a better understanding of parasite-endosymbiont interactions throughout the nematode life cycle.

Results: Over 215 million single-end 50 bp reads were generated from total RNA from D. immitis adult males and females, microfilariae ( $\mathrm{mf}$ ) and third and fourth-stage larvae (L3 and L4). We critically evaluated the transcriptomes of the various life cycle stages to reveal sex-biased transcriptional patterns, as well as transcriptional differences between larval stages that may be involved in larval maturation. Hierarchical clustering revealed both D. immitis and wDi transcriptional activity in the L3 stage is clearly distinct from other life cycle stages. Interestingly, a large proportion of both D. immitis and wDi genes display microfilarial-biased transcriptional patterns. Concurrent transcriptome sequencing identified potential molecular interactions between parasite and endosymbiont that are more prominent during certain life cycle stages. In support of metabolite provisioning between filarial nematodes and Wolbachia, the synthesis of the critical metabolite, heme, by wDi appears to be synchronized in a stage-specific manner (mf-specific) with the production of heme-binding proteins in D. immitis.
\end{abstract}

Conclusions: Our integrated transcriptomic study has highlighted interesting correlations between Wolbachia and D. immitis transcription throughout the life cycle and provided a resource that may be used for the development of novel intervention strategies, not only for the treatment and prevention of D. immitis infections, but of other closely related human parasites as well.

Keywords: Nematode, Filaria, Transcriptomics, Endosymbiosis, Wolbachia, RNA-seq

\footnotetext{
* Correspondence: michalsk@uwosh.edu

${ }^{3}$ Department of Biology and Microbiology, University of Wisconsin Oshkosh, Oshkosh, WI 54901, USA

Full list of author information is available at the end of the article
} 


\section{Background}

Dirofilaria immitis, the causative agent of canine heartworm disease, is a parasitic filarial nematode evolutionarily related to those responsible for human parasitic diseases such as lymphatic and cutaneous filariases. Like other onchocercids, $D$. immitis requires an arthropod vector for transmission (in this case Aedes, Anopheles or Culex mosquitoes), as well as a mammalian host. Natural patent infections of $D$. immitis occur in canids, including domestic dogs, coyotes and wolves, but can also occur in other mammals such as cats, ferrets and even humans [1]. The life cycle of $D$. immitis follows that of other filarial nematodes in that infected insects, mosquitoes in this case, introduce third-stage larvae (L3) into the vertebrate host during a blood meal. The L3 larvae molt first into fourthstage larvae (L4) and then adults within the vertebrate host. Adult males and females (AM and AF) residing in the pulmonary arteries of the mammalian host reproduce and give rise to microfilariae $(\mathrm{mf})$, which are released into the blood [2]. Circulating $\mathrm{mf}$ are ingested by a mosquito during another blood meal and molt twice within the vector before becoming infective L3 larvae [3].

If left untreated, adult worms present in the pulmonary vessels of the dog result in prolonged physical damage and inflammation. As the inflammation resolves and fibrosis occurs, affected vessels become less elastic and signs of right-sided heart failure can ensue [4]. Worms can also become lodged in the heart and pulmonary vessels where they can block circulation. Typically, the severity of disease is related to the number of adult worms present, although other factors, such as dog size and response to infection, have been hypothesized to affect severity $[1,4]$. Adult worms can be surgically removed with alligator foreceps, however, the currently recommended adulticidal treatment is the FDA-labeled adulticide melarsomine dihydrochloride, often used in conjunction with corticosteroids, aspirin and/or doxycycline [4]. While this drug is highly effective, treatment requires hospitalization with multiple intramuscular injections that can result in adverse side effects [4]. Furthermore, the relative cost of adulticidal treatment is typically greater than the cost of year-round monthly heartworm prevention. Adulticidal treatment is not recommended for use in cats, making prophylaxis the only option.

The development of heartworm disease is prevented by monthly administration of a macrocyclic lactone (i.e., ivermectin, selamectin, moxidectin or milbemycin oxime). These drugs kill susceptible L3s and L4s present in the animal. Elimination of these larval stages prevents the development of adult heartworms within the vertebrate host. As evidenced by estimated prevalence rates of up to $12.5 \%$ in the United States and much higher in other industrialized countries [5], there is, perhaps surprisingly, relatively poor compliance for domestic dogs. Factors shown to affect compliance include owner age, owner household income, and whether or not the pet is neutered [6]. Furthermore, resistance to current drugs is developing $[7,8]$. The few isolates rigorously tested for genotypes conferring resistance suggest P-glycoproteins are involved in providing resistance [9-11], however, the exact molecular mechanisms remain unclear at this time. The increase in reports of loss of efficacy/prophylaxis highlights the need for vaccine development against $D$. immitis and other filarial nematodes.

As with many other filarial nematodes, D. immitis contains an obligate bacterial endosymbiont, Wolbachia, that is present in the lateral chords of both sexes as well as the oocytes within the female reproductive tract. The nature of the essentiality of the Wolbachia-nematode symbiosis remains unclear, but, based on genomic sequences, is thought to derive from metabolite provisioning between the nematode and bacterium [12]. Early anti-Wolbachia treatments (doxycycline) in dogs were based on earlier observations from laboratory animals (jirds) where antibiotic treatments reduced parasite loads [13-16]. More recently, combined doxycycline and ivermectin treatment in dogs has been shown to have both adulticidal [17] and microfilaricidal activity in vivo [18], further demonstrating the essential mutualism between D. immitis and Wolbachia.

Genomic DNA sequences of the D. immitis and Wolbachia of $D$. immitis ( $w \mathrm{Di})$ genomes were recently completed and published [19]. While extremely useful, genomic approaches alone cannot provide a detailed understanding of the symbiotic relationship between nematode and bacterium. Related "omics" studies (transcriptomics/proteomics) are necessary to supply detailed functional information, enable improved diagnostics and provide new drug and vaccine targets. In light of developing drug resistance, we initiated the first series of concurrent transcriptional profiling experiments throughout the nematode life cycle to understand the global concerted transcriptional activity of the D. immitis and Wolbachia genomes and provide further insights into the evolutionary biology of these parasites and their symbionts.

\section{Methods}

\section{Parasites}

The $D$. immitis used in this study was from a naturally infected dog maintained at the University of Georgia College of Veterinary Medicine. This research was approved by the University of Georgia Institutional Animal Care and Use Committee.

Microfilariae were collected in whole blood drawn from the jugular vein of the infected dog. Microfilariae concentration was determined by viewing Giemsa stained thick blood smears. The $\mathrm{mf}$ concentration throughout the study was approximately $12,000 \mathrm{mf} / \mathrm{ml}$ of blood (data not shown). L3 were obtained by feeding microfilaremic blood to Aedes 
aegypti mosquitoes (black-eyed Liverpool strain) using artificial blood feeders, as previously described [20]. Fourteen days after feeding, L3 were obtained by gently crushing the infected mosquitoes, rinsing them onto a $32 \mu \mathrm{m}$ mesh sieve set in a petri dish and soaking them in warm Hanks' balanced salt solution, with larvae settling to the bottom of the dish for collection.

L4 were cultured from day 14 L3 isolated using the aforementioned procedure. Larvae were washed in phosphate buffered saline (Boston BioProducts) and cultured in 24 well plates (Becton Dickinson). Culture media consisting of RPMI-1640 (Lonza), heat inactivated fetal bovine serum (Sigma-Aldrich), gentamicin (Sigma-Aldrich), and penicillin/streptomycin combination (Hyclone Laboratories, Inc.) was changed every two days for fourteen days. Fully cast cuticles were observed beginning day 3 and lasted through day 14 of culture when L4 larvae were collected and frozen in $250 \mu \mathrm{l}$ of PBS. Microscopic examination of five randomly chosen worms confirmed that they were in the fourth larval stage (data not shown).

For collection of adult worms, the dog was necropsied and adult worms collected from the heart and pulmonary vessels. All worms were fast frozen in $1.5 \mathrm{~mL}$ graduated round bottom tubes (Eppendorf) at $-80^{\circ} \mathrm{C}$.

\section{Total RNA Isolation, Library Preparation and Sequencing}

D. immitis samples were homogenized with ceramic beads in CK14 tubes using a Minilys homogenizer (Precellys) and total RNA was extracted by organic extraction using Trizol (Ambion). Samples were treated with DNase I (Ambion) before further Trizol extraction and final purification. The RNA integrity, purity and concentration of all samples were assessed using a Bioanalyzer 2100 (Agilent Technologies). In order to capture $w$ Di transcripts, 100$125 \mathrm{ng}$ of total RNA and the NEBNext ${ }^{\circ}$ mRNA Library Prep Master Mix Set for Illumina ${ }^{\oplus}$ (Cat. \# E6110, New England Biolabs) or the NEBNext ${ }^{\circ}$ Ultra RNA Library Prep Kit for Illumina ${ }^{\oplus}$ (Cat. \# E7530, New England Biolabs) were used to prepare the libraries according to the kit instructions. Library quality was assessed using a DNA high sensitivity chip on a Bioanalyzer 2100 prior to sequencing. Transcriptomic libraries (50 bp single end reads) were sequenced on a Genome Analyzer IIx (Illumina). Two biological replicates $(\sim 18,500$ or 33,200 $\mathrm{mf} /$ replicate, $\sim 500 \mathrm{~L} 3 /$ replicate, 232 or $317 \mathrm{~L} 4 /$ replicate, 2 or $3 \mathrm{AF} /$ replicate and 4 or $5 \mathrm{AM} /$ replicate) were prepared and sequenced for each life cycle stage. No technical replicates were performed.

\section{Sequence Alignment and Differential Expression Analysis}

All data were analyzed using a local instance of Galaxy [21-23]. Sequence reads from each sample were analyzed using the Tuxedo protocol [24]. Briefly, RNA-Seq reads were first assessed for quality based on quality scores per base using FastQC [25]. RNA-Seq reads from each sample were aligned to the $D$. immitis genome (version 2.2) [19] using TopHat (v. 1.4.1) [26], a mapper capable of identifying splicing variants and junctions within eukaryotic transcriptomes. Default parameters were used. Reads aligned using TopHat were first viewed using the Integrated Genomics Viewer (IGV) [27] before being assembled into transcripts using Cufflinks (v. 2.1.1, default parameters). Cufflinks assemblies from all samples were merged using Cuffmerge and Cuffdiff was employed for differential expression testing. In Cuffdiff, quartile normalization and multi-read correct options were used. The false discovery rate (FDR) was set to 0.01 .

Similarly, the RNA-Seq reads from each sample were also mapped to the $w \mathrm{Di}$ genome (version 2.2) using Bowtie [28]. Reads aligned using Bowtie were assembled into transcripts using Cufflinks, then merged with Cuffmerge. Differential expression profiles were determined using Cuffdiff (v. 2.1.1). Default parameters for Cuffdiff were used except the minimum alignment count was set to 2 and FDR set to 0.01 .

Hierarchical clustering analysis was performed using Cluster 3.0 [29]. Mapped reads from biological replicates (BAM output files from either TopHat or Bowtie) were first merged then assembled into transcripts using Cufflinks. Normalized FPKM (Fragments Per Kilobase of transcript per Million mapped reads) values were hierarchically clustered using Pearson's uncentered correlation coefficient with a centroid linkage. Clustered data were depicted graphically (heatmap and dendrogram) using JavaTreeView [29]. GO terms were assigned to predicted $w$ Di gene models using InterProScan (version 4) [30,31]. Significantly enriched GO terms were identified using the web based Gene Ontology Enrichment Analysis Software Toolkit (GOEAST) [32] with the FDR set to 0.1.

\section{Results and discussion}

\section{Transcriptome overview}

In total, over 215 million single-end 50 bp reads were generated from total RNA from the $D$. immitis life cycle stages. Following the removal of low quality reads, approximately $55 \%$ of the sequenced reads mapped to the D. immitis reference genome (Table 1). Of the mappable reads, an average of $83.3 \% \pm 5.9 \%$ were uniquely mapped to the $D$. immitis genome, while the remaining $\sim 16.7 \%$ of reads (likely ribosomal RNA) mapped to multiple locations within the genome. Interestingly, FPKM distribution and coverage of the 12,857 predicted gene models varied greatly among the different $D$. immitis life cycle stages (Additional file 1: Dataset S1; Additional file 2: Figure S1A, Table 1). Although many reads map to rRNA, $\sim 80 \%$ of all $D$. immitis predicted gene models were expressed in every life cycle stage examined (Table 1). 
Table 1 Total number of reads sequenced and mapped to the $D$. immitis genome per $D$. immitis life cycle stage

\begin{tabular}{ccccccccccc}
\hline Sample & $\begin{array}{c}\text { Million reads } \\
\text { sequenced } \\
\text { (Rep 1) }\end{array}$ & $\begin{array}{c}\text { Million reads } \\
\text { mapped } \\
\text { (Rep 1) }\end{array}$ & $\begin{array}{c}\text { Million reads } \\
\text { sequenced } \\
\text { (Rep 2) }\end{array}$ & $\begin{array}{c}\text { Million reads } \\
\text { mapped } \\
\text { (Rep 2) }\end{array}$ & $\begin{array}{c}\text { Million reads } \\
\text { mapped (Reps } \\
\text { Combined) }\end{array}$ & $\begin{array}{c}\text { 50th } \\
\text { Percentile } \\
\text { (FPKM) }\end{array}$ & $\begin{array}{c}\text { 75th } \\
\text { Percentile } \\
\text { (FPKM) }\end{array}$ & $\begin{array}{c}\text { 95th } \\
\text { Percentile } \\
\text { (FPKM) }\end{array}$ & $\begin{array}{c}\text { Average } \\
\text { coverage (FPKM)/ } \\
\text { Transcript }\end{array}$ & $\begin{array}{c}\text { D. immitis } \\
\text { transcripts } \\
\text { detected (\%)** }\end{array}$ \\
\hline AM & 9.3 & 4.2 & 2.6 & 1.0 & 5.2 & 3.0 & 6.7 & 31.3 & 83.9 & 81 \\
AF & 14.8 & 7.2 & 1.4 & 0.5 & 7.7 & 7.3 & 14.9 & 53.4 & 92.0 & 93 \\
mf & $4.6^{*}$ & $1.6^{*}$ & 43.3 & 24.7 & 24.7 & 8.2 & 15.0 & 78.6 & 53.3 & 86 \\
L3 & 20.7 & 8.7 & 27.3 & 12.2 & 20.9 & 0.8 & 2.1 & 15.3 & 45.4 & 81 \\
L4 & 53.3 & 33.7 & 38.3 & 23.4 & 57.1 & 10.4 & 27.9 & 129.3 & 74.3 & 83 \\
\hline
\end{tabular}

Each life stage replicate, as well as the combined total of mapped reads per life stage are listed. *Due to DNA contamination, this replicate was omitted from further analysis. **Based on number of transcripts expressed (FPKM $>0$ ) per life cycle stage.

A pairwise comparison of replicates from each $D$. immitis life cycle stage can typically identify sampling or sequencing bias between the two biological replicates from each life cycle stage. As expected, most biological replicates are strikingly similar to one another (Additional file 2: Figure S1B-F). However, the $D$. immitis $\mathrm{mf}$ biological replicates displayed greater variation in gene expression than the other $D$. immitis life cycle stages (Additional file 2: Figure S1D). Closer interrogation revealed genomic DNA contamination of $D$. immitis $\mathrm{mf}$ biological replicate 1 (as indicated by reads mapping to intergenic regions in the $D$. immitis genome). Thus, for all further analysis, only data from $D$. immitis $\mathrm{mf}$ biological replicate 2 was utilized.

Because total RNA was used for library construction, the reads from the $D$. immitis life cycle stages (Table 1 ) were also mapped to the Wolbachia endosymbiont ( $w \mathrm{Di})$ genome. This approach was previously used to successfully sequence the transcriptome of Wolbachia from adult males and female gonads of the cattle parasite, Onchocerca ochengi ( $w \mathrm{Oo}$ ) [33]. As anticipated based on previous mixed transcriptomic studies [34], significantly fewer reads mapped to $w$ Di genes (Table 2 ) than to $D$. immitis genes (Table 1) in each life cycle stage: on average only $0.7 \%$ of sequenced reads (ranging from $0.02 \%$ to $2.11 \%$ depending on the life cycle stage) mapped to Wolbachia. A relatively high number of $w \mathrm{Di}$ transcripts (above 90\%) were detected by sequencing total RNA from $D$. immitis $\mathrm{AF}$ and $\mathrm{mf}$, while fewer transcripts were detected in the AM, L3 and L4 D. immitis samples ( 50-60\% of transcripts, Table 2). The extremely low representation of $w \mathrm{Di}$ reads is expected in a mixed transcriptome [34], nonetheless, the low coverage of the L4 sample is surprising, especially since numerous reports suggest that Wolbachia numbers rapidly increase during the L4 stage in filarial nematodes [1,35-37]. However, although Wolbachia read numbers in the L4 sample are low (especially compared to the relative nematode reads), the level of transcription (FPKM value) of certain genes in the L4 stages is relatively high compared to the other stages, suggesting that at least a certain subset of Wolbachia genes (and maybe more if the coverage were higher) are highly transcribed during the L4 stage. The variable and relatively low coverage of the $w$ Di transcriptome in some life cycle stages required certain parameters be adjusted (the minimum alignment count to be lowered) in order to conduct differential expression significance testing. Despite an overwhelming amount of host transcripts and rRNA, using these parameters, $653 w \mathrm{Di}$ genes were deemed differentially expressed $(\mathrm{q}<0.01)$ between at least two of the five life cycle stages (Additional file 3: Table S1).

\section{Stage-dependent transcriptional variation}

The pattern of similarity between $D$. immitis life cycle stages was addressed using hierarchical clustering. The resulting dendrogram (Figure $1 \mathrm{~A}$ ) reveals $D$. immitis AF and L4 larvae had the most similar transcriptional profiles, with AM and $\mathrm{mf}$ being more dissimilar and the L3 transcriptional profile was the most distinct (Figure 1A).

As was noted in the previous transcriptomic study of $B$. malayi life cycle stages [38], a large proportion of currently annotated $D$. immitis genes displayed significant

Table 2 Total number of reads mapped to the wDi genome per $D$. immitis life cycle stage

\begin{tabular}{cccccccc}
\hline Sample & $\begin{array}{c}\text { wDi Mapped } \\
\text { reads (50 bp) }\end{array}$ & $\begin{array}{c}\text { wDi Mapped } \\
\text { bases }\end{array}$ & $\begin{array}{c}\text { 50th Percentile } \\
\text { (FPKM) }\end{array}$ & $\begin{array}{c}\text { 75th Percentile } \\
\text { (FPKM) }\end{array}$ & $\begin{array}{c}\text { 95th Percentile } \\
\text { (FPKM) }\end{array}$ & $\begin{array}{c}\text { Average coverage } \\
\text { (FPKM)/Transcript }\end{array}$ & $\begin{array}{c}\text { wDi transcripts } \\
\text { detected (\%)* }\end{array}$ \\
\hline AM & 115,048 & $5,752,400$ & 91.0 & 215.4 & 1604.7 & 442.4 & 63 \\
AF & 340,598 & $17,029,900$ & 171.4 & 375.4 & 2194.9 & 463.5 & 93 \\
mf & 110,894 & $5,544,700$ & 1506.6 & 4047.7 & $22,418.4$ & 5141.4 & 287.3 \\
L3 & 26,673 & $1,333,650$ & 0 & 106.6 & 769.1 & 66 & 48 \\
L4 & 17,619 & 880,950 & 199.0 & 887.2 & 6480.2 & 1849.6 & 57 \\
\hline
\end{tabular}

*Based on number of transcripts expressed (FPKM > 0) per life cycle stage. 
Figure $1 D$. immitis life cycle transcriptome profiles. (A) Relationships between the various $D$. immitis life cycle stages (AM-Adult Male, AF-Adult Female, mf-microfilariae, L3-3rd stage larva, L4-4th stage larva) as revealed by hierarchical clustering. (B) Clustered transcriptomic data of D. immitis genes across the various life cycle stages. Only genes expressed in at least one stage are shown $(n=12,819)$. Each gene is represented by a single row. Data from biological replicates were combined prior to clustering. The color scale ranges from black (no expression) to red (very high expression). Black bars indicate the five clusters expressed predominantly in one D. immitis life cycle stage.

transcriptional variability (i.e., were significantly differentially expressed) among five life cycle stages studied herein (Additional file 4: Table S2). To identify groups of $D$. immitis genes preferentially transcribed during specific developmental stages, hierarchical clustering of all D. immitis genes was performed (Figure 1B). As indicated by the heatmap, each stage had at least one cluster of genes predominantly expressed in only that stage (Figure 1B). Based on this clustering, nearly $70 \%$ of D. immitis genes $(\mathrm{n}=8717)$ can be grouped into stageassociated transcriptional patterns (Additional file 5: Table S3) described in further detail below. Transferase activity (GO: 0016740) and glycolipid metabolic processes (GO: 0006664) are the only GO terms enriched among the remaining $\sim 30 \%$ of $D$. immitis genes which do not show any stage-associated transcriptional activity.

Previous cluster analysis of the predicted $D$. immitis nuclear proteome (version 1.3) with four other nematode proteomes (B. malayi, C. elegans, Trichinella sprialis and Ascaris suum) identified 850 'filarial-specific' proteins in clusters, i.e., conserved proteins uniquely shared by $D$. immitis and B. malayi, but lacking in the three other nematode species [19]. Comparison of these 850 predicted protein sequences with the improved version $2.2 \mathrm{D}$. immitis proteome (by BLASTp analysis) removed redundancies and yielded 834 predicted proteins which may represent common filarial nematode targets shared between $D$. immitis and B. malayi. Assessment of stage-specific transcription of these 834 gene products is listed in Additional file 6: Table S4 and summarized in Figure 2. Notably, only 367 of these genes have functional annotations, twenty of which are listed as hypothetical proteins. Hence, no functional information is available for over half of the 834 genes and many of the existing annotations are relatively vague. Nearly 65\% (539) were expressed in all life cycle stages examined while 25 of the 834 D. immitis and B. malayi specific genes were not expressed in any life cycle stage (Additional file 6: Table S4). The majority of genes (711) displayed no stage-associated transcriptional pattern. Conversely, 123 genes $(\sim 15 \%)$ were significantly upregulated in at least one life cycle stage (Additional file 6: Table S4, Figure 2). Of these 123 D. immitis genes with stage-associated transcriptional 


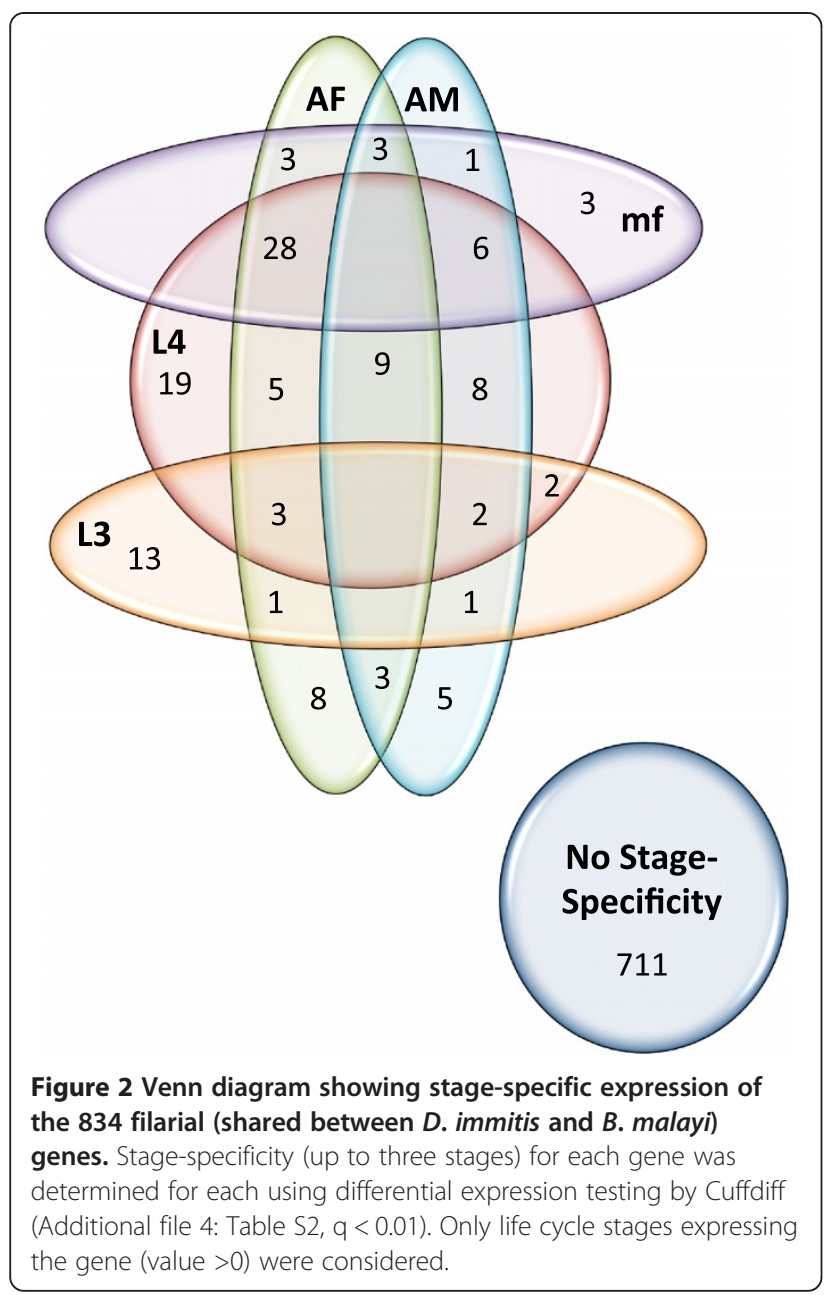

patterns, two-thirds were significantly upregulated in the L4 stage of D. immitis (alone or in conjunction with other life cycle stages, Figure 2) and include an apoptotic chromatin condensation inducer protein and a number of hypothetical proteins (Additional file 6: Table S4). Seven genes were mutually upregulated between the L3 and L4 stages. Among these 7 L3/L4 upregulated genes are a $227 \mathrm{kDa}$ spindle- and centromere-associated protein and a subunit of a nicotinic acetylcholine receptor. Fifteen additional genes are upregulated in the L3 stage, which may represent potential L3 targets, and include basement membrane proteoglycans and collagen domain containing proteins. Several genes (15) were upregulated in both AM and AF samples (Figure 2). These potential adulticidal targets include genes for adenylate kinase, a c-terminal binding protein and a bzip transcription factor family protein (Additional file 6: Table S4). An additional 48 genes were upregulated in AF (as well as other stages, Figure 2) including two hypothetical proteins, cell death specification protein 2 , an ecdysone induced protein and the ecdysone receptor, transcription of which was previously shown to be upregulated in D. immitis AF [39]. Upregulation of an additional 23 genes was observed for AM (as well as other stages, Figure 2) including a hypothetical protein and an RNA-binding domain containing protein (Additional file 6: Table S4).

\section{Gender-associated transcripts}

In the context of all five $D$. immitis life cycle stages examined, 414 transcripts and 502 transcripts display AM and AF-associated transcription, respectively (Figure 1B). $\mathrm{GO}$ enrichment analysis indicates functional categories such as phosphorylation and DNA repair are overrepresented among the AM associated genes (Additional file 7: Table S5). As expected, structural molecules related to male reproductive structures and sperm generation dominate the male-associated transcripts (Additional file 7: Table S5). These functionalities within D. immitis AMs strongly correlate with those previously identified in B. malayi [38]: structural molecule activity and proteins associated with protein phosphorylation were prominent among $B$. malayi male associated transcripts.

GO enrichment analysis revealed genes involved in cell division and protein/DNA complexes were overrepresented in D. immitis AF, as well as uterus development and chitin binding/processing, likely due to oocyte development (Additional file 7: Table S5). These findings differ from previous transcriptomic studies in B. malayi that identified enriched transcription factor activity, nuclear receptor activity, collagens/structural constituents of the cuticle and serpin activity in AF [38]. As described below, we observed enriched serpin transcripts in D. immitis $\mathrm{mf}$ and cuticle/collagens in D. immitis $\mathrm{mf}$, L3 and L4, not adult females. These differences may be attributable to experimental differences (the $B$. malayi life cycle transcriptome is based on only one sample, i.e., no biological replicates were run) or may represent differences between the two species.

A direct pairwise comparison of only $\mathrm{AM}$ and $\mathrm{AF}$ samples reveals that over 1400 genes display genderbiased transcriptional patterns, the majority $(\mathrm{n}=986)$ of which are upregulated in females. GO enrichment of the remaining 484 male-biased transcripts strongly reiterates the male-associated functional categories observed in the five-way comparison of life cycle stages (Additional file 8: Table S6). Once again, transcripts involved in protein phosphorylation, muscle development and structural molecule activity were prominent among the maleassociated transcripts (Additional file 8: Table S6). Given the increase in the number of female-biased transcripts in the pairwise comparison (986 vs. 502), it is interesting that fewer female-associated functional categories are overrepresented in the pairwise comparison with males (Additional file 8: Table S6) than the five-way comparison (Additional file 7: Table S5). The most prevalent over-represented functional categories among female- 
upregulated genes are those involved in development, including tissue development, neurogenesis and nervous system development (Additional file 8: Table S6). Other interesting female-biased functional categories include cell fate commitment/differentiation and sequencespecific DNA binding (Additional file 8: Table S6). Notably, many of these same gender-associated GO terms were enriched in a microarray gene expression study on adult B. malayi [40].

Interestingly, no $w \mathrm{Di}$ genes were significantly differentially expressed between AM and AFs. This is not entirely surprising since similar transcriptomic studies on $w$ Oo identified only 26 differentially expressed genes between AM and AF gonads (FDR of 0.05) [33].

\section{D. immitis larval development}

Within the context of gene expression among all five examined life cycle stages, a significant portion of $D$. immitis genes are preferentially transcribed in the L4 stage (4375 transcripts), whereas only 58 transcripts appear to be preferentially transcribed in the L3 stage (Figure 1B). However, genes associated with the extracellular matrix and structural components of the collagen/cuticle are significantly enriched among the L3-associated transcripts, and are likely involved in the L3 to L4 molt (Additional file 7: Table S5).

A number of biological processes are highlighted within the transcripts primarily expressed in the cultured L4 stage. Because larvae more than triple in length during the L4 stage [41], it is unsurprising that genes involved in development, more specifically cellular differentiation, larval development and growth predominate the L4 associated transcripts (Additional file 7: Table S5). Genes involved in reproduction and reproductive development were also significantly enriched within the L4-associated gene set. Additionally, prevalent among L4 transcripts (Additional file 7: Table S5) are genes involved in transcription/translation, muscle cell development and locomotion, cellular components and organization, cellular localization and migration, binding (protein/nucleotide/anion/heterocyclic), transport (endocytosis and secretion), apoptosis, pyrophosphatase activity, and cellular protein complex disassembly. Interestingly, $D$. immitis genes involved in multi-organism processes, or a biological process involving another organism of the same or different species, were enriched among the L4 associated gene set. This functional enrichment could suggest the symbiotic relationship between nematode and Wolbachia may be more prominent and potentially more targetable during this life cycle stage or is the result of reproductive development taking place in the L4 stage as the nematode prepares for sexual reproduction.

The L3 to L4 molt is a logical target for vaccine development with the goal of disrupting transmission as the nematode transitions from insect vector to mammalian host. Furthermore, immunization with irradiated L3 larvae from filarial nematodes produces robust and longlasting immunity [42-45]. Although we were unable to sequence the transcriptome of the L3 to L4 transition directly, direct comparison of the L3 and L4 stages may provide important information regarding transcriptional changes required to undergo this critical transition. Pairwise comparison of the mosquito-derived L3 and cultured L4 transcriptomes indicates that these larval transcriptional profiles are notably different. This is largely in contrast with previous results from $B$. malayi that showed the two larval stages displayed fairly similar transcriptional patterns [38]. Because the D. immitis L3 to L4 molt typically occurs faster than with $B$. malayi (3 days versus 8 days) $[41,46]$, this difference may represent species-specific transcriptional differences between the two filarial nematodes Alternatively, the transcriptional differences observed between L3 and L4 larvae of the two species may be the result of underlying experimental differences: the B. malayi L4 sample was derived in vivo, while the D. immitis L4 were cultured in vitro.

Examination of the L3/L4 comparison reveals 1450 significantly differentially expressed genes between L3 and L4 stage, (560 L3 upregulated, 890 L4 upregulated). Enriched GO terms among the L3 upregulated transcripts include genes involved in energy metabolism (including oxidoreductase activity), glycogen/carbohydrate synthesis, transport, muscle development, collagens and structural constituents of the cuticle and larval development (molting) (Additional file 9: Table S7). These results largely concur with the previous findings in B. malayi, where transcripts involved in glycogen biosynthesis, oxidoreductase activity, transport, collagen and structural constituents of the cuticle were enriched in the L3 stage [38]. However, the serpin activity and peptidase activity found to be enriched within the B. malayi L3 gene set were not identified as enriched functions within the $D$. immitis L3 upregulated gene set.

Functional categories overrepresented within the genes that are upregulated in the L4 stage (as compared to the L3 stage) are primarily involved in transcription and splicing, as well as larval development (Additional file 9: Table S7). These also include reproduction and reproductive tract development, meiosis, protein binding/complexes/ folding, endocytosis, nervous system development, as well as cellular component organization, a function also enriched within the L4 associated transcripts in the $B$. malayi transcriptome [38]. Notably, excretory-secretory proteins abundant during the $B$. malayi L3/L4 molt [47], including intermediate filament protein, thioredoxins, glutathione peroxidases, $\gamma$-glutamyl transpeptidases, macrophage migratory inhibitory factors and galectins display relatively high expression in the L3 and L4 stages of D. immitis. 


\section{Cathepsins and cystatins}

Cathepsins (cysteine proteases), more specifically the cathepsin $\mathrm{L}$ and $\mathrm{Z}$ families, of filarial nematodes are thought to be essential to a variety of biological processes. Deactivation of cathepsins, via either inhibitors or RNAi, drastically delayed or prevented L3 molting in D. immitis and $O$. volvulus [48-50]. Furthermore, RNAi knockdown of cathepsin L-like proteases (Bm-cpl-1 and Bm-cpl-5) and cathepsin Z-like protease (Bm-cpz-1) in B. malayi decreased AF fecundity [50]. Protein inhibitors of cysteine proteases, or cystatins, are another abundant larval protein family that may serve to control the developmental timing of cathepsin activity and initiation of molting. Due to their essentiality in critical developmental processes and expression patterns, cathepsins and cystatins have long been recognized as potential drug and vaccine targets. Recently, vaccination with a mutated cystatin from $B$. malayi significantly reduced the development of $B$. malayi L3 into adults in gerbils [51]. Moreover, a DNA vaccine with L. sigmodontis cysteine protease inhibitor-2 and ALT-1 (abundant larval transcript -1 ) significantly increased immunogenicity and reduced the number of adult worms and microfilaremia in a mouse model [52].

The $D$. immitis genome annotation contains 10 cathep$\sin \mathrm{L}$ family members, 2 cathepsin $\mathrm{Z}$ family members and 3 cystatin homologues. Overall, the $D$. immitis cathepsin L gene family products are highly expressed in the L3 stage and to a lesser extent in the L4 stage (Additional file 10: Figure S2). Likewise, expression of cystatins appears to correlate with the expression of cathepsin L proteins (highest in the L3 stage). Interestingly, the highest levels of expression for one cathepsin $\mathrm{Z}$ family member were observed in $\mathrm{mf}$, suggesting this cathepsin may play a role in molting within the vector host.

\section{Abundant larval transcripts}

Homologous proteins ALT-1 and ALT-2 (abundant larval transcripts -1 and -2 ) are predominantly expressed in the larval stages of $B$. malayi and were proposed as potential vaccine antigens due to their larval-specificity, high expression levels and lack of a human ortholog [53]. Stagespecific transcriptomic studies in B. malayi confirmed the presence of ALT-1 and ALT-2 in both larval stages examined (L3 and L4), however, transcript [38] and protein [47] levels of both were elevated in the L3 stage. The only orthologous protein in D. immitis, Di-20/22 L (nDi.2.2.2.g08197), originally identified as an L3 to L4 molt excretory-secretory protein [54], is highly expressed in the L3, L4 and $\mathrm{mf}$ stages (no significant difference in expression of Di-20/22 L is observed among any of the larval stages).

\section{D. immitis microfilarial transcriptome}

One ultimate objective in filarial research is the development of adulticidal therapies. While not the optimal target, drugs or vaccines that target the $\mathrm{mf}$ stage could complement existing treatments (especially in light of recent reduced efficacy reports) and serve as a strategy to limit transmission (particularly in highly endemic areas). In fact, previous studies have proven the feasibility of a vaccine based on mf-derived antigens [55-57]. A large proportion of $D$. immitis transcripts displayed microfilarial expression bias (3368) when compared to the other four stages examined (Figure 1B, Additional file 5: Table S3). Within this group of transcripts, GO terms related to transport are significantly overrepresented (Additional file 7: Table S5). More specifically, the transport of monovalent cations $\left(\mathrm{Na}^{+}\right.$and $\left.\mathrm{K}^{+}\right)$, oxygen and metal ions are overrepresented among mf-associated transcripts. Similar to findings observed in the transcriptomic studies on B. malayi microfilariae [38], nucleic acid binding and terms related to transcription are overrepresented among $D$. immitis mf-associated transcripts. Cuticle/collagen formation (procollagen-proline dioxygenase activity) was previously identified as overrepresented within B. malayi AF [38]. However, we observe enrichment of cuticle/collagens in the $D$. immitis microfilarial stage.

Interestingly, genes involved in eukaryotic cilium are significantly overrepresented in $D$. immitis $\mathrm{mf}$ transcripts (Additional file 7: Table S5). Electron microscopy studies first described 'unusual' cilia in the anterior portion of D. immitis microfilariae in 1968 [58]. The cilia are not limited to $D$. immitis $\mathrm{mf}$, but are found in other nematode species as well [59]. While unusual at the time, further structural studies indicated these ciliated structures were likely part of modified axon terminals of the amphids and phasmids, chemosensory organs of nematodes found in the anterior and posterior of the animal, respectively [60]. Elevated transcription levels of genes involved in the formation of these ciliated structures as well as the development of synaptic structures (Additional file 7: Table S5) suggests the amphids and/or phasmids are likely either developing or fully developed within the microfilarial stage of $D$. immitis and may therefore play a role in chemosensation and migration behavior.

Gamma-aminobutyric acid (GABA) signaling is another process enriched within $\mathrm{mf}$-associated $D$. immitis transcripts (Additional file 7: Table S5). Interestingly, the effectiveness of macrocyclic lactones such as ivermectin is derived from its agonistic activity of glutamate-gated $\mathrm{Cl}^{-}$channels [61] and $\mathrm{GABA}_{\mathrm{A}}$ receptors [62,63]. Mammalian GABAergic neurons are protected from ivermectin by the blood-brain-barrier and therefore relatively unaffected by the drug. However, ivermectin has been shown to cause bodywall and pharyngeal muscle paralysis in nematodes (especially in the larval stages) [61]. It is therefore not surprising that ivermectin administration prior to adulticidal treatment causes a rapid clearing of $\mathrm{mf}$ from dogs since $D$. immitis $\mathrm{mf}$ appear to 
contain especially high levels of these GABAergic receptors.

Binding, more specifically tetrapyrrole/heme binding, is functionally overrepresented among $D$. immitis microfilarial transcripts (Additional file 7: Table S5). As hemecontaining enzymes (e.g., cytochromes) are often associated with oxygen binding and metabolism, it is not surprising that oxygen binding was also overrepresented in $\mathrm{mf}$-associated transcripts. These findings are specifically interesting due to the proposed symbiotic provisioning of certain metabolites (such as heme and riboflavin) between Wolbachia and their obligate nematode hosts (described below) [12].

Another particularly interesting GO term overrepresented among $D$. immitis mf-associated transcripts was DNA integration (GO: 0015074, Additional file 7: Table S5), or the process in which a segment of DNA is incorporated into another, usually larger, DNA molecule such as a chromosome. As might be expected, the transcripts attributed to this GO term enrichment encode for integrases and pao retrotransposon peptidase family proteins. By transposing and propagating within the genome, retrotransposons possess the ability to disrupt essential genes within an organism. Many organisms, including B. malayi [64], have been shown to utilize small RNAs in order to counteract the effects of such transposons. However, unlike those in B. malayi, all pao type retrotranposons present in the $D$. immitis genome have been fragmented and thus inactivated [19]. Indeed, the D. immitis genome was the first sequenced metazoan genome completely lacking any functional transposable elements [19]. In the recent $B$. malayi life cycle transcriptomics study [38], the 4 (Bm1_18655, Bm1_21505, Bm1_36915 and Bm1_57480) of 28 annotated B. malayi retrotransposons that were actually transcribed were all transcribed within the microfilarial stage. Thus, the transcription of these inactivated pao retrotransposons in D. immitis (especially in the microfilarial stage) likely represents an evolutionary remnant.

Recently, the relationship between filarial nematodes and Wolbachia has been of great interest in the study of horizontal or lateral gene transfers (LGTs), where one organism acquires DNA from another organism. Indeed, it appears that widespread LGT has occurred from Wolbachia into their arthropod and nematode hosts $[65,66]$. Based on the genome sequences, it is estimated that $\sim 24 \%$ of the Wolbachia genome has been transferred to D. immitis [19]. However, only 9 of those candidate LGTs are potentially full-length gene transfers. This appears to differ significantly from $B$. malayi in which it is estimated that only $\sim 15 \%$ of the Wolbachia genome has been transferred to the nematode, but 36 of these LGTs appear to be full-length [66]. This phenomenon has not yet been studied in the context of the nematode/
Wolbachia life cycle, but it is interesting to postulate based on our transcriptomic results in D. immitis that LGTs may be more likely to occur during the mf stage when enzymes involved in this process (integrases) are highly expressed.

\section{Chitinase transcription}

Many studies have identified microfilarial-specific chitinase activity in filarial nematodes that release sheathed microfilariae (e.g., B. malayi, Wuchereria bancrofti and Loa loa) purportedly involved in exsheathment and/or penetration of the vector midgut $[67,68]$. Therefore, unsurprisingly, chitinase activity was previously identified as upregulated in B. malayi $\mathrm{mf}$ [38]. Conversely, filarial species that shed unsheathed $\mathrm{mf}$, including species such as D. immitis, Acanthocheilonema viteae and Onchocerca volvulus, have been shown to utilize a temperature specific $\left(37^{\circ} \mathrm{C}\right)$ L3-specific chitinase, homologous to the B. malayi mf-specific chitinase, to possibly degrade the cuticle during the L3 to L4 molt within the mammalian host. Of the three annotated chitinase genes within the D. immitis genome, the chitinase precursor protein (nDi.2.2.2.g01593) was consistently transcribed throughout the life cycle (Figure 3A). Although numerous studies have shown a lack of chitinase activity in D. immitis $\mathrm{mf}[67,68]$ and specific chitinase activity in the L3 stage [69,70], we observed significant upregulation of the cuticular endochitinase gene (nDi.2.2.2. g09584) in $\mathrm{mf}$ as compared to the L3 stage (Additional file 4: Table S2, Figure 3B). When describing the L3specific chitinase of $A$. viteae, Wu et al., identified a protein of similar molecular weight as the L3-specific chitinase present in $\mathrm{mf}$ that displayed the opposite temperature specificity (expression at $27^{\circ} \mathrm{C}$, not $37^{\circ} \mathrm{C}$ ) [69], suggesting the presence of an additional chitinase functional within the insect vector. Hence, there is some evidence to support our findings that expression of this endochitinase gene does occur in unsheathed microfilariae of certain nematode species. The exact role of endochitinase in unsheathed $\mathrm{mf}$ that do not require chitinases to infect the vector species $(D$. immitis $\mathrm{mf}$ do not penetrate the midgut of the insect vector but rather enter the Malpighian tubes through the posterior lumen of the vector midgut [71]), remains unclear. However, chitinase may be important to help the microfilariae migrate through the chitinous peritrophic membrane that surrounds the blood meal. Additionally, because L1 to L3 development for many filarial nematodes within the vector is intracellular, chitinase may be required to invade the host cell. As expected, expression levels of the chitinase gene (nDi.2.2.2.g09661) were much higher in the L3 stage (Figure $3 \mathrm{C}$ ) than any other chitinase-related gene (nDi.2.2.2.g01593 or $\mathrm{nDi}$.2.2.2.g09584) in any other life cycle stage (note difference in scales between Figure 3A/ $3 \mathrm{~B}$ and Figure $3 \mathrm{C})$. 
A

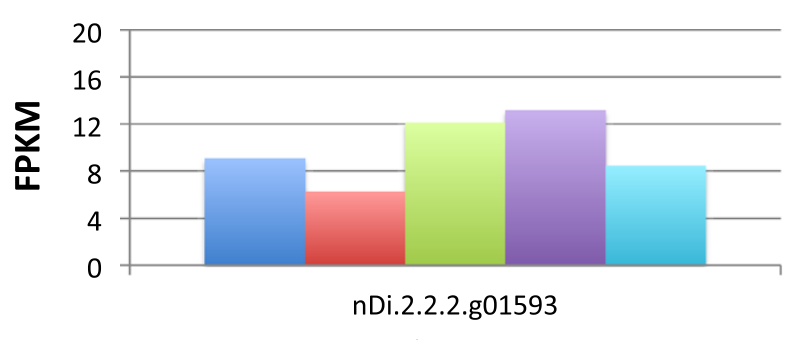

Chitinase

precursor protein

B

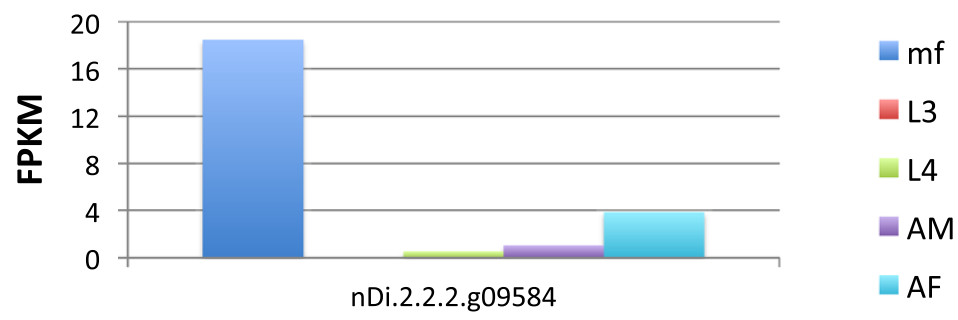

Cuticular

Endochitinase

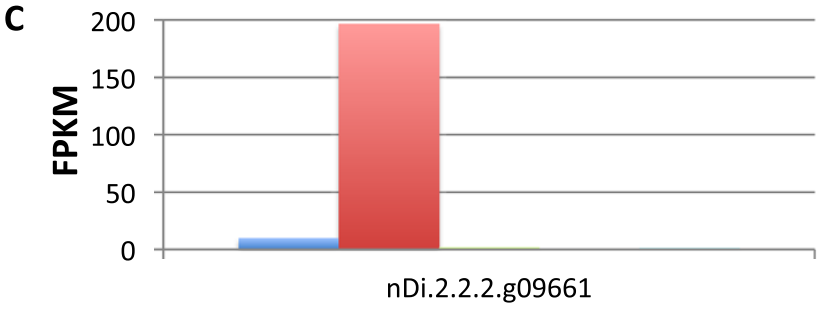

Chitinase

Figure 3 D. immitis chitinase expression. Expression profiles (FPKM values) of the D. immitis chitinase precursor protein (A), cuticular endochitinase (B) and chitinase (C) genes.

\section{Transcriptional profiles of the Wolbachia endosymbiont} (wDi)

Hierarchical clustering of transcript expression revealed the $w$ Di transcriptional profile of the L3 sample appears to be quite different from the other life cycle stages (Additional file 11: Figure S3A). Interestingly, the resulting heatmap (Additional file 11: Figure S3B) clearly shows comparatively high expression of a number of $w \mathrm{Di}$ genes during the $\mathrm{mf}$ stage in comparison to the other life cycle stages. This may therefore indicate increased transcriptional activity occurs in Wolbachia during the $\mathrm{mf}$ stage relative to other life cycle stages. Likewise, although very low $w \mathrm{Di}$ transcriptome coverage was observed in the L4 stage (Table 2), a significant portion of genes appear to be highly transcribed (Additional file 11: Figure S3B), suggesting Wolbachia transcription may be substantial during this stage. Although stage-associated gene clusters were identified (Additional file 11: Figure S3B, Additional file 12: Table S8), no functional categories (GO terms) were enriched among any life cycle stage. However, transcripts involved in certain processes were frequently associated with a distinct life cycle stage, e.g., in accordance with previous reports suggesting that Wolbachia numbers rapidly increase during the L4 stage in filarial nematodes [1,35-37], a number of critical cell division proteins fall within the L4 cluster (Additional file 12: Table S8). Godel et al. [19] suggested a number of $w \mathrm{Di}$ genes that may be suitable drug targets. The development of drugs that disrupt particular life cycle stages, especially adults and L4s, would be of interest. Specific targets that have been suggested include proteins involved in nucleic acid synthesis (DnaB), enzymes involved in fatty acid synthesis (FabZ and AcpS) [19] and the previously identified anti-Wolbachia target, FtsZ, a cell division protein [72]. Our $w$ Di transcriptomic data revealed that these potential drug targets all exhibit preferential expression in the $\mathrm{mf}$ stage, but also displayed low levels of expression in both adult males and females (Additional file 13: Table S9). Interestingly, expression of only one (DnaB) or two (DnaB and FtsZ) of these potential Wolbachia targets was detected for the L3 and L4 stages, respectively. 
The $w$ Di transcriptomes of $\mathrm{mf}$ and L3 were the most significantly different from one another and produced 254 differentially expressed genes, all of which were upregulated in $\mathrm{mf}$. Notably, $w \mathrm{Di}$ genes including $m u r F$, mraY, murG and murJ (involved in peptidoglycan synthesis), the gene for hemB (porphobilinogen synthase, part of the heme biosynthetic pathway), a gene involved in pyrimidine biosynthesis (dihydroorotate dehydrogenase) and virB9 (one component of the type 4 secretion system) are all upregulated in mf compared to the L3 stage and detailed further below. Among the 107 genes differentially expressed between the L3 and L4 stages, genes involved in peptidoglycan synthesis (murF, murG and murJ), heme biosynthesis (hemB), the type 4 secretion system (virB6 and virD4), the Sec translocase (SecF and $\mathrm{SecD}$ ), and riboflavin biosynthesis (ribA) were all upregulated in the L4 stage compared to the L3 stage (Additional file 3: Table S1). Conversely, the heme biosynthesis gene, hemE, and a component of the twin-arginine transporter $(\operatorname{tat} A)$ were upregulated in the L3 stage compared to the L4 stage (Additional file 3: Table S1). Among the 188 genes differentially expressed between $\mathrm{mf}$ and L4 stages were DNA recombination and mismatch repair proteins (RmuC and MutL), virB11 (type 4 secretion system protein), yajC (Sec translocase), tat $A$ and hemE (Additional file 3: Table S1), all of which were upregulated in the $\mathrm{mf}$ stage compared to the L4 stage.

While the host-symbiont relationship between filarial nematodes and Wolbachia cannot be reduced to one process or factor, a number of obvious relationships can be inferred based on genetic profiling of the two organisms $[12,73,74]$. D. immitis lacks specific genes required for de novo synthesis of purines, pyrimidines and other cofactors (heme and riboflavin). Similar to Wolbachia from B. malayi $(w \mathrm{Bm})$ [12] these pathways are complete within the $w \mathrm{Di}$ genome [19]. Conversely, biosynthetic pathways of other vitamins and cofactors such as Coenzyme A, NAD, biotin, lipoic acid, ubiquinone and pyridoxal phosphate are incomplete in $w \mathrm{Di}$ and thus, may be supplied to the endosymbiont by the host. Although previous transcriptomic studies found little evidence of metabolite provisioning between Wolbachia and O. ochengi [33], we focused our analysis on transcription of these specific $w$ Di pathways (Figure 4, Additional file 13: Table S9), which may provide further information on the evolutionary biology of Wolbachia and highlight opportunities for further drug targeting and development.

\section{Heme synthesis}

Serving as an essential cofactor in a number of critical biological processes, heme is a virtually indispensable part of life [75]. The iron-containing tetrapyrrole is synthesized via a highly conserved enzymatic pathway. Similar to B. malayi $[12,76]$, genome sequencing of $D$. immitis and $w$ Di revealed that, like other nematodes, $D$. immitis lacked homologues for nearly every enzymatic step in the heme biosynthetic pathway, while $w \mathrm{Di}$ retained an intact and plausibly functional heme pathway [19]. Although all D. immitis life cycle stages display at least low levels of expression for most genes in the pathway, the entire $w \mathrm{Di}$ heme biosynthesis pathway was predominantly expressed in the mf stage (Figure 4). This is an especially intriguing result given that tetrapyrrole/heme binding was functionally overrepresented in the $D$. immitis $\mathrm{mf}$ transcriptome (described above, Additional file 7: Table S5). Moreover, the fact that feedback inhibition does not appear to be affecting transcriptional levels of genes in the beginning of the pathway (e.g. hemA) [75,77], as well as high levels of expression of the $\mathrm{CcmB}$ heme exporter in the microfilarial stage (Figure 4) suggest the possibility that heme, while being synthesized in Wolbachia, may indeed be exported to the nematode host in a stage-specific manner. The suggestion that heme requirements may be high during the $\mathrm{mf}$ stage is particularly interesting given that $\mathrm{mf}$ typically survive for long periods of time in the blood of the mammalian host, where heme is seemingly plentiful. However, this stockpiling of heme may be related to a lack of heme availability in the upcoming insect vector stages of parasite development. Recent investigations have found that the capability of Plasmodium spp to synthesize heme is critical for parasite development in the mosquito stages [78], suggesting that heme availability may be limiting within the mosquito. A similar scenario may exist for filarial nematode development: microfilariae may be accumulating stores of heme prior to mosquito-induced heme deprivation. The potential for this Wolbachia-nematode metabolite provisioning to be stage-specific in filarial worms as our data suggests warrants further investigation.

\section{Riboflavin synthesis}

The synthesis of riboflavin, a precursor to flavin moieties often utilized as enzyme cofactors, is another critical biosynthetic pathway retained in the $w \mathrm{Di}$ genome and absent in the D. immitis genome [12]. Interestingly, the riboflavin synthesis pathway appears complete and functional within $w \mathrm{Bm}$ and $w \mathrm{Di}$, but with the exception of one gene (ribA) has been completely pseudogenized in $w$ Oo [33]. Again, although transcription of the pathway was evident in nearly every life cycle stage, all genes in the pathway were expressed at relatively high levels in $\mathrm{mf}$ stage (Figure 4). RibA and RibE are also highly expressed in the L4 stage, while low levels of transcription for all other genes in the riboflavin pathway were transcribed in the L4 stage (Figure 4). The $w$ Di transcriptomic data differ slightly in comparison to previous $w \mathrm{Bm}$ qRT-PCR data where expression of ribA was greatest in the L3 stage and lowest in the $\mathrm{mf}$ stage [79]. 


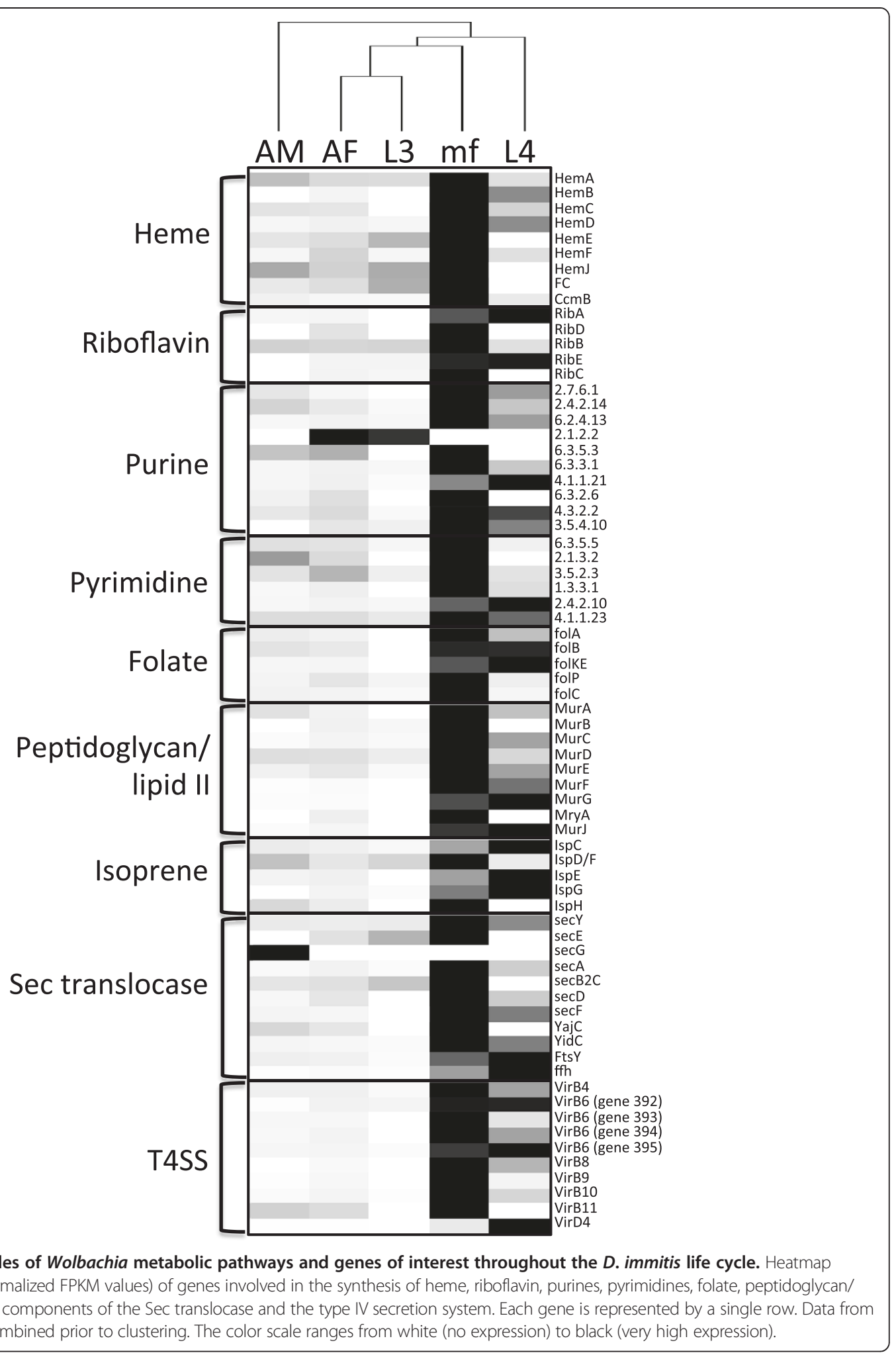

\section{Nucleotide synthesis}

De novo nucleotide synthesis pathways are often lost in endosymbiotic organisms [12], but are maintained in $w \mathrm{Di}$ [19] and may be used to supplement the host nucleotide pool. Transcripts involved in the $w \mathrm{Di}$ de novo purine biosynthesis pathway are prominently expressed in the $\mathrm{mf}$ stage and the L4 stage (Figure 4), with the exception of one gene (phosphoribosylglycinamide formyltransferase, 
E.C. 2.1.2.2, fig_82301.12.peg.508), for which expression was detected only in AF and L3 samples. All genes in the pyrimidine de novo synthesis pathway are highly transcribed in the $\mathrm{mf}$ stage and to a lesser extent in the adult stages (Figure 4). Although no transcription of the second step (E.C. 2.1.3.2) in the pyrimidine synthesis pathway is observed for either larval stage (L3 or L4), the final two enzymatic steps within the pathway are highly expressed in the L4 stage (Figure 4). Interestingly, in agreement with previous reports suggesting Wolbachia proliferate within the L4 stage of filarial nematodes [1,35-37], it appears that both nucleotide biosynthesis pathways in $w$ Di are highly transcribed in the L4 stage. However, transcription of nucleotide biosynthesis genes is also highly elevated in the $\mathrm{mf}$ stage, when relatively little Wolbachia replication is occurring $[35,36]$. Hence, while transcription of $w$ Di nucleotide biosynthesis genes in the L4 stage appears to correlate with Wolbachia DNA synthesis, the increased transcription of these nucleotide biosynthesis pathways in the $\mathrm{mf}$ stage (when Wolbachia numbers remain low within the nematode) suggests stage-specific supplementation of the D. immitis nucleotide pool by Wolbachia.

\section{Folate synthesis}

Folate synthesis is intricately linked to a number of critical cofactors and metabolites including heme and purine biosynthesis, as well as methylation of DNA [80]. Interestingly, $w \mathrm{Bm}$ cannot synthesize folate due to a lack of the first two enzymes in the pathway (FolA and FolB) [12]. However, both $w \mathrm{Di}$ and $w \mathrm{Oo}$ have retained these genes and can likely synthesize folate $[19,33]$. The $w \mathrm{Di}$ life cycle transcriptome revealed the entire pathway was transcribed in every life cycle stage examined except for the L3 stage. The entire 5-step pathway is apparently highly expressed in $\mathrm{mf}$ stage, while the two intermediate steps (FolB and FolKE) were most highly expressed in L4 stage (Figure 4).

\section{Lipid II synthesis}

Because members of the Wolbachia genus maintain the genes necessary for lipid II synthesis (Mur operon) within their reduced genomes [73,81], yet lack genes required to synthesize peptidoglycan, the cell wall structure/ components may be very different in Wolbachia compared to other bacteria. Additionally, recent evidence suggests that this pathway is functional in Wolbachia [82] and may play a role in cell division [81], thus making it a worthwhile anti-Wolbachia drug target. Once again, based on our transcriptomics data, evidence of complete expression of this $w \mathrm{Di}$ pathway exists only in AF and mf stages, (Figure 4). Although transcription of certain genes within this pathway is apparently quite high in the L4 stage and to a lesser extent in AMs, no transcripts for $\operatorname{murB}$ and mra $Y$ genes were detected in these stages. Interestingly, the enzyme responsible for the formation of lipid I (MraY), was only detected in mf and AFs while MurG, the enzyme responsible for the conversion of lipid I to lipid II, was detected in every stage except for L3. Other genes likely involved in the formation of the pentapeptide moiety of peptidoglycans include metC (cystathione betalyase, fig_82301.12.peg.810) and $d d l$ (D-alanine-D-alanine ligase, fig_82301.12.peg.613). The exact composition of the pentapeptide component of Wolbachia peptidoglycan remains unknown, however the presence of $d d l$ in the reduced genome strongly suggests the incorporation of D-isomer amino acids. Wolbachia were originally thought to lack genes for the racemases necessary to produce D-isomers from L-isomers (typically L-alanine or Lglutamate). However, although classically recognized as a component of the methionine biosynthesis pathway, recombinant MetC from $w \mathrm{Bm}$ was recently shown to have in vitro non-canonical L-alanine racemase activity [83] and likely has a role in peptidoglycan synthesis in Wolbachia. Similar to genes in the Mur operon, transcript levels of these two genes, $d d l$ and metC, are highest in the $\mathrm{mf}$ and L4 stages. Likewise, transcript levels of the peptidoglycan lipid II flippase, MurJ, thought to be involved in flipping peptidoglycan chains across the membrane into the periplasm, were significantly higher in the L4 and $\mathrm{mf}$ stages (Additional file 3: Table S1, Figure 4).

\section{Isoprenoid synthesis}

Isoprenoids are critical metabolites synthesized by all living organisms via two possible pathways: the mevalonic acid (MVA) pathway, utilized by yeast and animals $[84,85]$ or the methylerythritol 4-phosphate (MEP) pathway present in most bacteria, protozoa and algae [86]. With the exception of plants, most organisms exclusively utilize only one of these pathways for isoprenoid precursor biosynthesis [87]. The genome of $w \mathrm{Bm}$ revealed the presence of a nearly complete MEP pathway (all but the gene for 1-deoxy-D-xyulose-5-phosphate synthase), for the biosynthesis of isoprenoids [12]. Likewise, $w \mathrm{Di}$ also lacks the enzyme responsible for the first step in the MEP pathway. As previously suggested for $w \mathrm{Bm}[12]$, it is possible the missing gene-product (1-deoxy-D-xylulose-5phosphate) is supplied to $w \mathrm{Di}$ by $D$. immitis. The MEP pathway of Wolbachia, which is absent in humans and nematodes, exemplifies an ideal target for the development of specific anti-Wolbachia therapies. Our transcriptomic data suggests that although some genes in the pathway were expressed at considerably higher levels in the L4 than other stages (IspC, IspE and IspG), transcription of other steps in the pathway are extremely low (IspD/F and IspH) (Figure 4). Likewise, expression of various genes in the pathway was completely absent in the L3 (IspE and IspH) and AM stages (IspG). This apparent lack of transcription may simply be the result of the extremely 
low $w \mathrm{Di}$ transcriptome coverage reported in some stages (Table 2). Expression of all genes involved in the $w \mathrm{Di}$ isoprenoid biosynthetic pathway was detected in the AF and $\mathrm{mf}$ stages, albeit at a lower frequency than those preferentially expressed in the L4 stage.

\section{Wolbachia secretion systems}

Wolbachia protein secretion systems impart a molecular mechanism by which the bacteria may interact with the nematode host. The Sec protein translocation system is responsible for the translocation of unfolded protein across the cell membrane into the periplasmic space. Transmembrane proteins, SecY and SecE, form the core of the protein-translocating channel. A third transmembrane protein, SecG associates with SecYE to form a heterotrimeric complex. Although not required for translocation, the addition of SecG, to form SecYEG, stimulates translocation through the channel at low temperatures $\left(20^{\circ} \mathrm{C}\right)$ [88]. SecE, SecY and SecA (the ATPase providing the energy for translocation) have been shown to be absolutely essential to bacterial viability, making the Sec translocase an attractive anti-Wolbachia drug target $[88,89]$. Interestingly, $w$ Di from $D$. immitis L4s only expressed the SecY portion of the transmembrane complex (Figure 4). The non-essential SecG was only expressed in wDi from AMs. Additionally, SecY is expressed in $w \mathrm{Di}$ from AM samples, however SecE was not expressed in this stage (Figure 4). With the exception of SecG (which as aforementioned is non-essential and was only expressed in AM), every gene in the $w \mathrm{Di}$ Sec pathway was transcribed in D. immitis mf. This supports a functional $\mathrm{Sec}$ translocation system in the $\mathrm{mf}$ stage. The driving force for protein translocation via the Sec pathway, SecA, was expressed in every life cycle stage examined while transcripts for $\mathrm{SecB}$, a chaperone that targets proteins to the translocation complex, were detected in every stage except L4 (Figure 4).

An accessory complex (SecDFyajC) comprised of three additional proteins $(\mathrm{SecD}, \mathrm{SecF}$ and YajC) interacts with SecYEG [88]. Curiously, the exact role of the SecDFyajC complex is not clear. It is not required for protein transport in vitro however, in vivo protein transport is severely affected by a lack of either SecD or SecF [88]. All three components of the $w$ Di SecDFyajC complex were transcribed in AM, AF and $\mathrm{mf}$ stages of $D$. immitis, while only the two essential proteins of this complex (SecD and SecF) were expressed in the L4 stage. Interestingly, Wolbachia from the third larval stage (L3) of D. immitis did not express any part of this complex (SecDFyajC, Figure 4).

Other Sec translocase accessory proteins include: YidC which interacts with Sec translocase and is involved in membrane insertion of both Sec-dependent and Secindependent proteins [88]; a protein component of the signal recognition particle, ffh, that targets certain unfolded proteins to the membrane for export; and FtsY the proposed receptor for the signal recognition particle [89]. Expression of these three accessory proteins was detected in every $D$. immitis life cycle stage tested, but particularly high in the L4 stage (Figure 4).

The Sec-independent twin arginine translocation (Tat) protein translocation system is found in most bacteria and generally comprised of multiple protein components. While thought to be essential to protein transport via the Tat system [90], Wolbachia lack the tatB gene, but like other $\alpha$-proteobacteria likely maintain a functional TatAC translocase [91]. Translational fusion experiments in $E$. coli revealed TatA is the most highly expressed Tat component [92] and expected to be at a 40:1 molar ratio with TatC [90]. Expression of $w$ Di tatA (fig_82301.12. peg.349) was highest in the L3 stage, less in the AM, AF and $\mathrm{mf}$ stages and undetected in the L4 stage. Alternatively, tatC (fig_82301.12.peg.851) was highly expressed in $\mathrm{mf}$ and L4 stage, less in AM and AF stages and not at all in the L3 stage (Additional file 13: Table S9). The FPKM values for the $D$. immitis AM, AF and $\mathrm{mf}$ stages suggest a 5-24 fold higher expression of $w$ Di tatA over tatC. The observed lack of expression of $w \mathrm{Di}$ tatC in the L3 stage may simply be due to transcript levels being extremely low and beyond the threshold of detection.

Secretion by Wolbachia requires translocation not only across the plasma membrane into the periplasm (via the Sec or Tat systems described above), but additionally transport across the outer membrane is required. The multi-subunit type IV secretion system (T4SS) is highly conserved and evolutionarily maintained in Wolbachia from insects, arthropods and filarial nematodes [79,93,94]. Likely due to an intracellular lifestyle, Wolbachia express a minimally functional T4SS lacking four pilus-associated proteins (VirB1, B2, B5 and B7). The remaining eight constituents of the T4SS are clustered within the genome: one cluster comprised of genes encoding VirB3, B4 and B6; the other cluster encodes VirB8, B9, B10, B11 and VirD4 $[94,95]$. Similar to $w \mathrm{Bm}[79]$, the VirB8-VirD4 operon in $w \mathrm{Di}$ (82301.12.peg.724-728) is preceded by the ribA gene (82310.12.peg.723, Figure 4), the first enzyme in riboflavin biosynthesis. Genes for VirB4 and four VirB6 components constitute the other T4SS operon (82301.12.peg.391-395), however, the gene for virB3 was absent in the most recent annotation of the $w \mathrm{Di}$ genome (version 2.2). Closer examination reveals virB3 appeared in the first version of the $w$ Di genome (83201.4.peg.46), but the relatively small gene was omitted from the second annotation. Multiple reads from our transcriptome data mapped to this region of the genome (wDi22.scaf1:777977-778268), validating the location and transcription of virB3 (Additional file 14: Figure S4). All components of the $w \mathrm{Di}$ T4SS are highly expressed in D. immitis $\mathrm{mf}$, and to a lesser extent in AFs (Figure 4). All other life cycle stages (AM, L3 and L4) lacked transcription of one or multiple critical components 
of the T4SS (Additional file 13: Table S9). Interestingly, D. immitis L4s exhibited high transcription levels of all $w$ Di T4SS components except for virB11, an ATPase required for assembly of the T4SS, for which no expression was detected in this stage. It is noteworthy that the Wolbachia Sec translocase, the Tat translocation system and the type IV secretion system were all highly expressed in $D$. immitis $\mathrm{mf}$, especially since the majority of Wolbachia proteins previously detected in $B$. malayi excretory-secretory products were of microfilarial origin [47].

One interesting attribute of the T4SS is its ability to mediate not only protein transport, but nucleic acid transfer as well $[94,96]$. Possibly such an ability to transport DNA is involved in LGT from Wolbachia to the nematode host. Interestingly, in another potential stagespecific host-endosymbiont interaction, genes involved in DNA integration were upregulated in D. immitis $\mathrm{mf}$ transcripts (Additional file 7: Table S5) while the T4SS appears to be highly transcribed in Wolbachia from $D$. immitis mfs. Again, while simply conjecture at this point, it is interesting to postulate that LGTs may be more likely to occur during the $\mathrm{mf}$ stage since LGTpromoting transcripts are expressed in both organisms at this stage.

\section{Conclusions}

Stage-specificity of host-symbiont interactions has been demonstrated in entomopathogenic nematodes and their associated enterobacterial symbionts [97], but has been largely unreported in filarial nematodes [33]. Ideally, future therapeutic strategies will target both $D$. immitis and Wolbachia and therefore require a greater spatial and temporal understanding of parasite-endosymbiont relationship. This dynamic mixed-transcriptome study adds to the growing body of literature on filarial nematode gene expression and has revealed interesting correlations between Wolbachia and D. immitis throughout the nematode life cycle. Moreover, this data provides an invaluable resource with which to develop improved therapeutic strategies for $D$. immitis and potentially other closely related filarial nematodes.

\section{Availability of supporting data}

The mapped reads are available in the NCBI short read archive accession SRP048819.

\section{Additional files}

Additional file 1: Dataset S1. Transcript FPKM values for Dirofilaria immitis life cycle transcriptome.

Additional file 2: Figure S1. (A) Distribution of D. immitis gene densities (FPKM coverage) for each D. immitis life cycle stage (biological replicates grouped). Pairwise comparison of D. immitis AM (B), AF (C), mf
(D), L3 (E) and L4 (F) biological replicates. Each point represents a single gene.

Additional file 3: Table S1. List of significantly $(q<0.01)$ differentially expressed $w D i$ genes.

Additional file 4: Table S2. List of significantly $(q<0.01)$ differentially expressed D. immitis genes.

Additional file 5: Table S3. Stage-associated D. immitis gene lists. Additional file 6: Table S4. Stage-Specific Expression of the 834 D. immitis Genes Uniquely Shared with B. malayi.

Additional file 7: Table S5. List of over-represented GO terms for stage-associated $D$. immitis genes.

Additional file 8: Table S6. List of over-represented GO terms for direct AM and AF pairwise comparison.

Additional file 9: Table S7. List of overrepresented GO terms for direct L3 and L4 pairwise comparison.

Additional file 10: Figure S2. D. immitis cathepsin and cystatin expression. Expression profiles (FPKM values) of cathepsin $L$ and $Z$ family members, as well as cysteine protease inhibitors (cystatins).

Additional file 11: Figure S3. (A) Hierarchical clustering reveals relationships between Wolbachia transcription profiles during the various D. immitis life cycle stages. (B) Clustered transcriptomic data of wDi genes across the various life cycle stages. Only genes expressed in at least one stage are shown. Each gene is represented by a single row. Data from biological replicates were combined prior to clustering. The color scale ranges from black (no expression) to red (very high expression).

Additional file 12: Table S8. Hierarchical clustering of wDi expression throughout $D$. immitis life cycle reveals stage-associated (as indicated by X) wDi genes.

Additional file 13: Table S9. FPKM values for $w D i$ transcriptome throughout $D$. immitis life cycle stages.

Additional file 14: Figure S4. IGV visualization of transcriptomic reads mapping to the putative location of $w D i$ virB3. The selected region spans the gap between the wDi lysyl-tRNA synthetase gene (82301.12.peg.390) and the wDi virB4 gene (82301.12.peg.391), where the virB3 gene annotation was omitted from version 2.2 of the wDi genome. Reads mapped to this region of the genome (wDi22.scaf1:777977-778268), are indicated (blue bars) for each D. immitis biological replicate).

\section{Abbreviations}

AF: Adult female; ALT: Abundant larval transcript; AM: Adult male; D. immitis: Dirofilaria immitis; FDR: False discovery rate; FPKM: Fragments Per Kilobase of transcript per Million mapped reads; GABA: Gamma-aminobutyric acid; GO: Gene ontology; IGV: Integrated Genomics Viewer; L3: Third-stage larvae; L4: Fourth-stage larvae; LGT: Lateral gene transfer; mf: microfilariae; MEP: Methylerythritol 4-phosphate; MVA: Mevalonic acid; Tat: Twin arginine translocase; T4SS: Type 4 secretion system; wBm: Wolbachia from Brugia malayi; wDi: Wolbachia from Dirofilaria immitis; wOo: Wolbachia from Onchocerca ochengi.

\section{Competing interests}

The authors declare that they have no competing interests.

\section{Author's contributions}

Conceived and designed the experiments: ANL JMF ARM BES MLM. Performed the experiments and analyzed the data: ANL CCE MDR MLM. Drafted the manuscript: ANL BES ARM. All authors read, edited and approved the final manuscript.

\section{Acknowledgements}

We thank Dr. Mark Blaxter for providing the list of 850 protein clusters uniquely shared between D. immitis and B. malayi, Dr. William Kozek for helpful comments, Dr. Sanjay Kumar and Dr. Laurence Ettwiller for bioinformatic support and Dr. Donald Comb, Dr. William Jack, Dr. Clotilde Carlow and James Ellard for their continued scientific support. This work was funded by New England Biolabs, Inc. 


\section{Author details}

${ }^{1}$ New England Biolabs, Inc., Genome Biology Division, 240 County Road, Ipswich, MA 01938, USA. ²Department of Infectious Diseases, University of Georgia, College Veterinary Medicine, 501 D. W. Brooks Drive, Athens, GA 30602, USA. ${ }^{3}$ Department of Biology and Microbiology, University of Wisconsin Oshkosh, Oshkosh, WI 54901, USA.

Received: 25 August 2014 Accepted: 14 November 2014 Published: 29 November 2014

\section{References}

1. McCall JW, Genchi C, Kramer LH, Guerrero J, Venco L: Heartworm disease in animals and humans. Adv Parasitol 2008, 66:193-285.

2. Kotani T, Powers KG: Developmental stages of Dirofilaria immitis in the dog. Am J Vet Res 1982, 43(12):2199-2206.

3. Taylor AE: The development of Dirofilaria immitis in the mosquito Aedes aegypti. J Helminthol 1960, 34:27-38.

4. Bowman DD, Atkins CE: Heartworm biology, treatment, and control. Vet Clin North Am Small Anim Pract 2009, 39(6):1127-1158. vii.

5. Lee AC, Montgomery SP, Theis $\mathrm{JH}$, Blagburn BL, Eberhard ML: Public health issues concerning the widespread distribution of canine heartworm disease. Trends Parasitol 2010, 26(4):168-173.

6. Brown HE, Harrington LC, Kaufman PE, McKay T, Bowman DD, Nelson CT, Wang D, Lund R: Key factors influencing canine heartworm, Dirofilaria immitis, in the United States. Parasit Vectors 2012, 5:245.

7. Wolstenholme AJ, Fairweather I, Prichard R, Von Samson-Himmelstjerna G, Sangster NC: Drug resistance in veterinary helminths. Trends Parasitol 2004, 20(10):469-476.

8. Kaplan RM: Drug resistance in nematodes of veterinary importance: a status report. Trends Parasitol 2004, 20(10):477-481.

9. Geary TG, Bourguinat C, Prichard RK: Evidence for macrocyclic lactone anthelmintic resistance in Dirofilaria immitis. Top Companion Anim Med 2011, 26(4):186-192.

10. Bourguinat C, Keller K, Bhan A, Peregrine A, Geary T, Prichard R: Macrocyclic lactone resistance in Dirofilaria immitis. Vet Parasitol 2011, 181(2-4):388-392.

11. Bourguinat C, Keller K, Blagburn B, Schenker R, Geary TG, Prichard RK: Correlation between loss of efficacy of macrocyclic lactone heartworm anthelmintics and P-glycoprotein genotype. Vet Parasitol 2011, 176(4):374-381.

12. Foster J, Ganatra M, Kamal I, Ware J, Makarova K, Ivanova N, Bhattacharyya A, Kapatral V, Kumar S, Posfai J, Vincze T, Ingram J, Moran L, Lapidus A, Omelchenko M, Kyrpides N, Ghedin E, Wang S, Goltsman E, Joukov V, Ostrovskaya O, Tsukerman K, Mazur M, Comb D, Koonin E, Slatko B: The Wolbachia genome of Brugia malayi: endosymbiont evolution within a human pathogenic nematode. PLoS Biol 2005, 3(4):e121.

13. Bosshardt SC, McCall JW, Coleman SU, Jones KL, Petit TA, Klei TR: Prophylactic activity of tetracycline against Brugia pahangi infection in jirds (Meriones unguiculatus). J Parasitol 1993, 79(5):775-777.

14. Bandi C, McCall JW, Genchi C, Corona S, Venco L, Sacchi L: Effects of tetracycline on the filarial worms Brugia pahangi and Dirofilaria immitis and their bacterial endosymbionts Wolbachia. Int J Parasitol 1999, 29(2):357-364

15. Gasser RB, Cantacessi C: Heartworm genomics: unprecedented opportunities for fundamental molecular insights and new intervention strategies. Top Companion Anim Med 2011, 26(4):193-199.

16. McCall JW: What is the rationale for the doxycyclin dose of $10 \mathrm{mg} / \mathrm{kg}$ twice daily for one month in the American Heartworm Society Guidelines for treatment of heartworm-infected dogs? American Heartworm Society Bulletin 2014, 41(1):8-9.

17. Kramer L, Grandi G, Leoni M, Passeri B, McCall J, Genchi C, Mortarino M, Bazzocchi C: Wolbachia and its influence on the pathology and immunology of Dirofilaria immitis infection. Vet Parasitol 2008, 158(3):191-195.

18. Bazzocchi C, Mortarino M, Grandi G, Kramer LH, Genchi C, Bandi C, Genchi M, Sacchi L, McCall JW: Combined ivermectin and doxycycline treatment has microfilaricidal and adulticidal activity against Dirofilaria immitis in experimentally infected dogs. Int J Parasitol 2008, 38(12):1401-1410.

19. Godel C, Kumar S, Koutsovoulos G, Ludin P, Nilsson D, Comandatore F, Wrobel N, Thompson M, Schmid CD, Goto S, Bringaud F, Wolstenholme A, Bandi C, Epe C, Kaminsky R, Blaxter M, Maser P: The genome of the heartworm, Dirofilaria immitis, reveals drug and vaccine targets. Faseb $\mathrm{J}$ 2012, 26(11):4650-4661.

20. McCall JW: The role of arthropods in the development of animal models for filariasis research. J Georgia Entomol Soc 1981, 16:283-293.

21. Giardine B, Riemer C, Hardison RC, Burhans R, Elnitski L, Shah P, Zhang Y, Blankenberg D, Albert I, Taylor J, Miller W, Kent W, Nekrutenko A: Galaxy: a platform for interactive large-scale genome analysis. Genome Res 2005, 15(10):1451-1455.

22. Blankenberg D, Von Kuster G, Coraor N, Ananda G, Lazarus R, Mangan M, Nekrutenko A, Taylor J: Galaxy: a web-based genome analysis tool for experimentalists. Curr Protoc Mol Biol 2010, Chapter 19(Unit 19 10):11-21.

23. Goecks J, Nekrutenko A, Taylor J: Galaxy: a comprehensive approach for supporting accessible, reproducible, and transparent computational research in the life sciences. Genome Bio/ 2010, 11(8):R86.

24. Trapnell C, Williams BA, Pertea G, Mortazavi A, Kwan G, Van Baren MJ, Salzberg SL, Wold BJ, Pachter L: Transcript assembly and quantification by RNA-Seq reveals unannotated transcripts and isoform switching during cell differentiation. Nat Biotechnol 2010, 28(5):511-515.

25. FastQC: a quality control tool for high throughput sequence data. http://www.bioinformatics.babraham.ac.uk/projects/fastqc

26. Trapnell C, Pachter L, Salzberg SL: TopHat: discovering splice junctions with RNA-Seq. Bioinformatics 2009, 25(9):1105-1111.

27. Thorvaldsdottir H, Robinson JT, Mesirov JP: Integrative Genomics Viewer (IGV): high-performance genomics data visualization and exploration. Brief Bioinform 2013, 14(2):178-192.

28. Langmead B, Trapnell C, Pop M, Salzberg SL: Ultrafast and memory-efficient alignment of short DNA sequences to the human genome. Genome Biol 2009, 10(3):R25.

29. Eisen MB, Spellman PT, Brown PO, Botstein D: Cluster analysis and display of genome-wide expression patterns. Proc Natl Acad Sci U S A 1998, 95(25):14863-14868

30. Zdobnov EM, Apweiler R: InterProScan-an integration platform for the signature-recognition methods in InterPro. Bioinformatics 2001, 17(9):847-848

31. Quevillon E, Silventoinen V, Pillai S, Harte N, Mulder N, Apweiler R, Lopez R InterProScan: protein domains identifier. Nucleic Acids Res 2005, 33(Web Server issue):W116-W120.

32. Zheng Q, Wang XJ: GOEAST: a web-based software toolkit for Gene Ontology enrichment analysis. Nucleic Acids Res 2008, 36(Web Server issue):W358-W363.

33. Darby AC, Armstrong SD, Bah GS, Kaur G, Hughes MA, Kay SM, Koldkjaer P, Rainbow L, Radford AD, Blaxter ML, Tanya VN, Trees AJ, Cordaux R, Wastling JM, Makepeace BL: Analysis of gene expression from the Wolbachia genome of a filarial nematode supports both metabolic and defensive roles within the symbiosis. Genome Res 2012, 22(12):2467-2477.

34. Kawahara $Y$, Oono $Y$, Kanamori $H$, Matsumoto $T$, Itoh T, Minami E: Simultaneous RNA-seq analysis of a mixed transcriptome of rice and blast fungus interaction. PLoS One 2012, 7(11):e49423.

35. Fenn K, Blaxter M: Quantification of Wolbachia bacteria in Brugia malayi through the nematode lifecycle. Mol Biochem Parasitol 2004 137(2):361-364

36. McGarry HF, Egerton GL, Taylor MJ: Population dynamics of Wolbachia bacterial endosymbionts in Brugia malayi. Mol Biochem Parasitol 2004, 135(1):57-67.

37. Fischer K, Beatty WL, Jiang D, Weil GJ, Fischer PU: Tissue and stage-specific distribution of Wolbachia in Brugia malayi. PLoS Negl Trop Dis 2011, 5(5):e1174

38. Choi YJ, Ghedin E, Berriman M, McQuillan J, Holroyd N, Mayhew GF, Christensen BM, Michalski ML: A deep sequencing approach to comparatively analyze the transcriptome of lifecycle stages of the filarial worm. Brugia malayi PLoS Negl Trop Dis 2011, 5(12):e1409.

39. Shea C, Richer J, Tzertzinis G, Maina CV: An EcR homolog from the filarial parasite, Dirofilaria immitis requires a ligand-activated partner for transactivation. Mol Biochem Parasitol 2010, 171(2):55-63.

40. Li BW, Rush AC, Jiang DJ, Mitreva M, Abubucker S, Weil GJ: Gender-associated genes in filarial nematodes are important for reproduction and potential intervention targets. PLoS Negl Trop Dis 2011, 5(1):e947.

41. Mutafchiev Y, Bain O, Williams Z, McCall JW, Michalski ML: Intraperitoneal development of the filarial nematode Brugia malayi in the Mongolian jird (Meriones unguiculatus). Parasitol Res 2014, 113(5):1827-1835. 
42. Storey DM, Al-Mukhtar AS: Vaccination of Jirds, Meriones unguiculatus, against Litomosoides carinii and Brugia pahangi using irradiate larvae of L. carinii. Tropenmed Parasitol 1982, 33(1):23-24.

43. Mejia JS, Carlow CK: An analysis of the humoral immune response of dogs following vaccination with irradiated infective larvae of Dirofilaria immitis. Parasite Immunol 1994, 16(3):157-164.

44. Le Goff L, Martin C, Oswald IP, Vuong PN, Petit G, Ungeheuer MN, Bain O: Parasitology and immunology of mice vaccinated with irradiated Litomosoides sigmodontis larvae. Parasitology 2000, 120(Pt 3):271-280.

45. Babayan SA, Attout T, Harris A, Taylor MD, Le Goff L, Vuong PN, Renia L, Allen JE, Bain O: Vaccination against filarial nematodes with irradiated larvae provides long-term protection against the third larval stage but not against subsequent life cycle stages. Int J Parasitol 2006, 36(8):903-914.

46. Bain O, Casiraghi M, Martin C, Uni S: The nematoda Filarioidea: critical analysis linking molecular and traditional approaches. Parasite 2008, 15(3):342-348.

47. Bennuru S, Semnani R, Meng Z, Ribeiro JM, Veenstra TD, Nutman TB: Brugia malayi excreted/secreted proteins at the host/parasite interface: stage- and gender-specific proteomic profiling. PLOS Negl Trop Dis 2009, 3(4):e410

48. Richer JK, Hunt WG, Sakanari JA, Grieve RB: Dirofilaria immitis: effect of fluoromethyl ketone cysteine protease inhibitors on the third- to fourth-stage molt. Exp Parasitol 1993, 76(3):221-231.

49. Lustigman S, McKerrow JH, Shah K, Lui J, Huima T, Hough M, Brotman B: Cloning of a cysteine protease required for the molting of Onchocerca volvulus third stage larvae. J Biol Chem 1996, 271(47):30181-30189.

50. Ford L, Zhang J, Liu J, Hashmi S, Fuhrman JA, Oksov Y, Lustigman S: Functional analysis of the cathepsin-like cysteine protease genes in adult Brugia malayi using RNA interference. PLOS Negl Trop Dis 2009, 3(2):e377.

51. Arumugam S, Wei J, Ward D, Abraham D, Lustigman S, Zhan B, Klei TR: Vaccination with a genetically modified Brugia malayi cysteine protease inhibitor-2 reduces adult parasite numbers and affects the fertility of female worms following a subcutaneous challenge of Mongolian gerbils (Meriones unguiculatus) with B. malayi infective larvae. Int J Parasitol 2014.

52. Babayan SA, Luo H, Gray N, Taylor DW, Allen JE: Deletion of parasite immune modulatory sequences combined with immune activating signals enhances vaccine mediated protection against filarial nematodes. PLoS Negl Trop Dis 2012, 6(12):e1968.

53. Gregory WF, Atmadja AK, Allen JE, Maizels RM: The abundant larval transcript-1 and -2 genes of Brugia malayi encode stage-specific candidate vaccine antigens for filariasis. Infect Immun 2000, 68(7):4174-4179

54. Frank GR, Grieve RB: Metabolic labeling of Dirofilaria immitis third- and fourth-stage larvae and their excretory-secretory products. J Parasitol 1991, 77(6):950-956.

55. Townson S, Bianco AE: Immunization of calves against the microfilariae of Onchocerca lienalis. J Helminthol 1982, 56(4):297-303.

56. Makepeace BL, Jensen SA, Laney SJ, Nfon CK, Njongmeta LM, Tanya VN, Williams SA, Bianco AE, Trees AJ: Immunisation with a multivalent, subunit vaccine reduces patent infection in a natural bovine model of onchocerciasis during intense field exposure. PLoS Negl Trop Dis 2009, 3(11):e544.

57. Ziewer S, Hubner MP, Dubben B, Hoffmann WH, Bain O, Martin C, Hoerauf A, Specht S: Immunization with L. sigmodontis microfilariae reduces peripheral microfilaraemia after challenge infection by inhibition of filarial embryogenesis. PLoS Neg/ Trop Dis 2012, 6(3):e1558.

58. Kozek WJ: Unusual cilia in the microfilaria of Dirofilaria immitis. J Parasitol 1968, 54(4):838-844.

59. Kozek WJ, Raccurt C: Ultrastructure of Mansonella ozzardi microfilaria, with a comparison of the South American (Simuliid-transmitted) and the Carribean (Culicoid-transmitted) forms. Tropenmed Parasitol 1983, 34:38-53.

60. Kozek WJ: Ultrastructure of the mcrofilaria of Dirofilaria immitis. J Parasitol 1971, 57(5):1052-1067.

61. Crump A, Omura S: Ivermectin, 'wonder drug' from Japan: the human use perspective. Proc Jpn Acad Ser B Phys Biol Sci 2011, 87(2):13-28.

62. Adelsberger $\mathrm{H}$, Lepier $\mathrm{A}$, Dudel J: Activation of rat recombinant alpha(1) beta(2)gamma(2S) GABA(A) receptor by the insecticide ivermectin. Eur J Pharmacol 2000, 394(2-3):163-170.

63. Dawson GR, Wafford KA, Smith A, Marshall GR, Bayley PJ, Schaeffer JM, Meinke PT, Mckernan RM: Anticonvulsant and adverse effects of avermectin analogs in mice are mediated through the $\mathrm{\gamma}$-aminobutyric acid A receptor. J Pharmacol Exp Ther 2000, 295:1051-1060.

64. Poole CB, Davis PJ, Jin J, McReynolds LA: Cloning and bioinformatic identification of small RNAs in the filarial nematode. Brugia malayi $\mathrm{Mol}$ Biochem Parasitol 2010, 169(2):87-94.

65. Dunning Hotopp JC, Clark ME, Oliveira DC, Foster JM, Fischer P, Munoz Torres MC, Giebel JD, Kumar N, Ishmael N, Wang S, Ingram J, Nene RV, Shepard J, Tomkins J, Richards S, Spiro DJ, Ghedin E, Slatko BE, Tettelin H, Werren $\mathrm{JH}$ : Widespread lateral gene transfer from intracellular bacteria to multicellular eukaryotes. Science 2007, 317(5845):1753-1756.

66. Ioannidis P, Johnston KL, Riley DR, Kumar N, White JR, Olarte KT, Ott S, Tallon LJ, Foster JM, Taylor MJ, Dunning Hotopp JC: Extensively duplicated and transcriptionally active recent lateral gene transfer from a bacterial Wolbachia endosymbiont to its host filarial nematode Brugia malayi. BMC Genomics 2013, 14:639.

67. Wu Y, Preston G, Bianco AE: Chitinase is stored and secreted from the inner body of microfilariae and has a role in exsheathment in the parasitic nematode Brugia malayi. Mol Biochem Parasitol 2008, 161:55-62.

68. Adam R, Kaltmann B, Rudin W, Friedrich T, Marti T, Lucius R: Identification of chitinase as the immunodominant filarial antigen recognized by sera of vaccinated rodents. J Biol Chem 1996, 271(3):1441-1447.

69. Wu Y, Adam R, Williams SA, Bianco AE: Chitinase genes expressed by infective larvae of the filarial nematodes, Acanthocheilonema viteae and Onchocerca volvulus. Mol Biochem Parasitol 1996, 75(2):207-219.

70. Wu Y, Egerton G, Underwood AP, Sakuda S, Bianco AE: Expression and secretion of a larval-specific chitinase (family 18 glycosyl hydrolase) by the infective stages of the parasitic nematode. Onchocerca volvulus J Biol Chem 2001, 276(45):42557-42564.

71. Fuhrman JA: Filarial Chtinases. Parasitol Today 1995, 11(7):259-261.

72. Li Z, Garner AL, Gloeckner C, Janda KD, Carlow CK: Targeting the Wolbachia cell division protein FtsZ as a new approach for antifilarial therapy. PLoS Negl Trop Dis 2011, 5(11):e1411.

73. Dunning Hotopp JC, Lin M, Madupu R, Crabtree J, Angiuoli SV, Eisen JA, Seshadri R, Ren Q, Wu M, Utterback TR, Smith S, Lewis M, Khouri H, Zhang C, Niu H, Lin Q, Ohashi N, Zhi N, Nelson W, Brinkac LM, Dodson RJ, Rosovitz MJ, Sundaram J, Daugherty SC, Davidsen T, Durkin AS, Gwinn M, Haft DH, Selengut JD, Sullivan SA, et al: Comparative genomics of emerging human ehrlichiosis agents. PLoS Genet 2006, 2(2):e21.

74. Brownlie JC, Adamski M, Slatko B, McGraw EA: Diversifying selection and host adaptation in two endosymbiont genomes. BMC Evol Biol 2007, 7:68.

75. Hamza I, Dailey HA: One ring to rule them all: trafficking of heme and heme synthesis intermediates in the metazoans. Biochim Biophys Acta 2012, 1823(9):1617-1632.

76. Wu B, Novelli J, Foster J, Vaisvila R, Conway L, Ingram J, Ganatra M, Rao AU, Hamza I, Slatko B: The heme biosynthetic pathway of the obligate Wolbachia endosymbiont of Brugia malayi as a potential anti-filarial drug target. PLoS Negl Trop Dis 2009, 3(7):e475.

77. Sheftel $A D$, Mason $A B$, Ponka P: The long history of iron in the Universe and in health and disease. Biochim Biophys Acta 2012, 1820(3):161-187.

78. Nagaraj VA, Sundaram B, Varadarajan NM, Subramani PA, Kalappa DM, Ghosh SK, Padmanaban G: Malaria parasite-synthesized heme is essential in the mosquito and liver stages and complements host heme in the blood stages of infection. PLOS Pathog 2013, 9(8):e1003522.

79. Li Z, Carlow CK: Characterization of transcription factors that regulate the type IV secretion system and riboflavin biosynthesis in Wolbachia of Brugia malayi. PLoS One 2012, 7(12):e51597.

80. Lienhart WD, Gudipati V, Macheroux P: The human flavoproteome. Arch Biochem Biophys 2013, 535(2):150-162.

81. Henrichfreise B, Schiefer A, Schneider T, Nzukou E, Poellinger C, Hoffmann TJ, Johnston KL, Moelleken K, Wiedemann I, Pfarr K, Hoefauf A, Shi HG: Functional conservation of the lipid II biosynthesis pathway in the cell wall-less bacteria Chlamydia and Wolbachia: why is lipid II needed? Mol Microbiol 2009, 73(5):913-923.

82. Bennuru S, Meng Z, Ribeiro JM, Semnani RT, Ghedin E, Chan K, Lucas DA, Veenstra TD, Nutman TB: Stage-specific proteomic expression patterns of the human filarial parasite Brugia malayi and its endosymbiont Wolbachia. Proc Natl Acad Sci U S A 2011, 108(23):9649-9654.

83. Vollmer J, Schiefer A, Schneider T, Julicher K, Johnston KL, Taylor MJ, Sahl HG, Hoerauf A, Pfarr K: Requirement of lipid II biosynthesis for cell division in cell wall-less Wolbachia, endobacteria of arthropods and filarial nematodes. Int J Med Microbiol 2013, 303(3):140-149. 
84. Chappell J: Biochemistry and molecular biology of the isoprenoid biosynthetic pathway in plants. Annu Rev Plant Physiol Plant Mol Biol 1995, 46:521-547.

85. McGarvey DJ, Croteau R: Terpenoid metabolism. Plant Cell 1995, 7(7):1015-1026

86. Rohmer M: The discovery of a mevalonate-independent pathway for isoprenoid biosynthesis in bacteria, algae and higher plants. Nat Prod Rep 1999, 16(5):565-574.

87. Rodriguez-Concepcion M, Boronat A: Elucidation of the methylerythritol phosphate pathway for isoprenoid biosynthesis in bacteria and plastids. A metabolic milestone achieved through genomics. Plant Physiol 2002, 130(3):1079-1089.

88. Veenendaal AK, van der Does $C$, Driessen AJ: The protein-conducting channel SecYEG. Biochim Biophys Acta 2004, 1694(1-3):81-95

89. Mori H, Ito K: The Sec protein-translocation pathway. Trends Microbiol 2001, 9(10):494-500.

90. Buchanan G, De Leeuw E, Stanley NR, Wexler M, Berks BC, Sargent F Palmer T: Functional complexity of the twin-arginine translocase TatC component revealed by site-directed mutagenesis. Mol Microbiol 2002, 43(6):1457-1470.

91. Nunez PA, Soria M, Farber MD: The twin-arginine translocation pathway in alpha-proteobacteria is functionally preserved irrespective of genomic and regulatory divergence. PLoS One 2012, 7(3):e33605.

92. Jack RL, Sargent F, Berks BC, Sawers G, Palmer T: Constitutive expression of Escherichia coli tat genes indicates an important role for the twin-arginine translocase during aerobic and anaerobic growth. J Bacteriol 2001, 183(5):1801-1804.

93. Masui S, Sasaki T, Ishikawa H: Genes for the type IV secretion system in an intracellular symbiont, Wolbachia, a causative agent of various sexual alterations in arthropods. J Bacterio/ 2000, 182(22):6529-6531.

94. Pichon S, Bouchon D, Cordaux R, Chen L, Garrett RA, Greve P: Conservation of the Type IV secretion system throughout Wolbachia evolution. Biochem Biophys Res Commun 2009, 385(4):557-562.

95. Rances E, Voronin D, Tran-Van V, Mavingui P: Genetic and functional characterization of the type IV secretion system in Wolbachia. J Bacteriol 2008, 190(14):5020-5030.

96. Christie PJ, Whitaker N, Gonzalez-Rivera C: Mechanism and structure of the bacterial type IV secretion systems. Biochim Biophys Acta 2014.

97. Koppenhofer HS, Gaugler R: Defensive Mutualism in Microbial Symbiosis. Boca Raton, FL: CRC Press; 2010.

doi:10.1186/1471-2164-15-1041

Cite this article as: Luck et al:: Concurrent transcriptional profiling of Dirofilaria immitis and its Wolbachia endosymbiont throughout the nematode life cycle reveals coordinated gene expression. BMC Genomics 2014 15:1041.

\section{Submit your next manuscript to BioMed Central and take full advantage of:}

- Convenient online submission

- Thorough peer review

- No space constraints or color figure charges

- Immediate publication on acceptance

- Inclusion in PubMed, CAS, Scopus and Google Scholar

- Research which is freely available for redistribution 